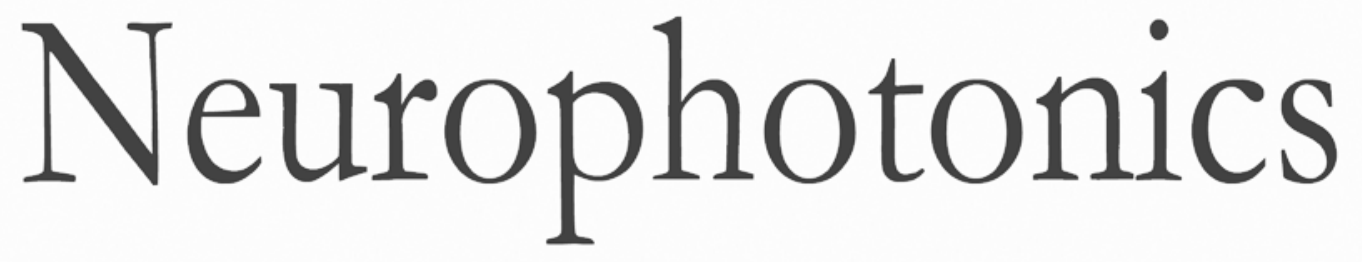

Motion tracking and electromyography-assisted identification of mirror hand contributions to functional nearinfrared spectroscopy images acquired during a finger-tapping task performed by children with cerebral palsy

Nathan Hervey

Bilal Khan

Laura Shagman

Fenghua Tian

Mauricio R. Delgado

Kirsten Tulchin-Francis

Angela Shierk
Heather Roberts

Linsley Smith

Dahlia Reid

Nancy J. Clegg

Hanli Liu

Duncan MacFarlane

George Alexandrakis 


\title{
Motion tracking and electromyography-assisted identification of mirror hand contributions to functional near-infrared spectroscopy images acquired during a finger-tapping task performed by children with cerebral palsy
}

\author{
Nathan Hervey, a,* Bilal Khan,, ${ }^{a}$ Laura Shagman, ${ }^{\mathrm{b}}$ Fenghua Tian,, Mauricio R. Delgado, ${ }^{\mathrm{c}}$ Kirsten Tulchin-Francis, ${ }^{\mathrm{c}}$ \\ Angela Shierk, ${ }^{c}$ Heather Roberts, ${ }^{c}$ Linsley Smith, ${ }^{c}$ Dahlia Reid, ${ }^{c}$ Nancy J. Clegg, ${ }^{c}$ Hanli Liu, ${ }^{a}$ Duncan \\ MacFarlane, ${ }^{b}$ and George Alexandrakis ${ }^{a}$ \\ aUniversity of Texas at Arlington and University of Texas Southwestern Medical Center at Dallas, Joint Graduate Program in \\ Biomedical Engineering, 500 UTA Boulevard, Arlington, Texas 76010, United States \\ bUniversity of Texas at Dallas, 800 West Campbell Road, Richardson, Texas 75080, United States \\ 'Scottish Rite Hospital for Children, Department of Neurology, 2222 Welborn Street, Dallas, Texas 75219, United States
}

\begin{abstract}
Recent studies have demonstrated functional near-infrared spectroscopy (fNIRS) to be a viable and sensitive method for imaging sensorimotor cortex activity in children with cerebral palsy (CP). However, during unilateral finger tapping, children with $\mathrm{CP}$ often exhibit unintended motions in the nontapping hand, known as mirror motions, which confuse the interpretation of resulting fNIRS images. This work presents a method for separating some of the mirror motion contributions to fNIRS images and demonstrates its application to fNIRS data from four children with CP performing a finger-tapping task with mirror motions. Finger motion and arm muscle activity were measured simultaneously with fNIRS signals using motion tracking and electromyography (EMG), respectively. Subsequently, subject-specific regressors were created from the motion capture or EMG data and independent component analysis was combined with a general linear model to create an fNIRS image representing activation due to the tapping hand and one image representing activation due to the mirror hand. The proposed method can provide information on how mirror motions contribute to fNIRS images, and in some cases, it helps remove mirror motion contamination from the tapping hand activation images. () The Authors. Published by SPIE under a Creative Commons Attribution 3.0 Unported License. Distribution or reproduction of this work in whole or in part requires full attribution of the original publication, including its DOI. [DOI: 10.1117/1.NPh.1.2.025009]
\end{abstract}

Keywords: cerebral palsy; functional near-infrared spectroscopy; general linear model; independent component analysis.

Paper 14054PR received Jun. 27, 2014; revised manuscript received Sep. 22, 2014; accepted for publication Oct. 14, 2014; published online Nov. 11, 2014.

\section{Introduction}

Cerebral palsy $(\mathrm{CP})$ is a heterogeneous group of disorders, the unifying feature of which is an impairment of fine and gross motor function due to dysgenesis or injury in early brain development. ${ }^{1}$ In patients with $\mathrm{CP}$, motor function does not necessarily predict or represent the underlying physiology. Therefore, observation of neurological activation patterns would help create a more coherent understanding of how brain function relates to motor control deficits. ${ }^{2}$ Sensorimotor activation patterns of the brain in children with $\mathrm{CP}$ have been mapped by functional magnetic resonance imaging (fMRI) in several recent studies. ${ }^{3-6}$ However, fMRI requires subjects to remain still for extended periods of time in a restricted space, which is particularly difficult to do when working with children with CP. Recently, functional near-infrared spectroscopy (fNIRS) has been demonstrated as a feasible alternate neuroimaging technique that enables brain activation measurements under relatively unrestricted conditions. $^{7}$ fNIRS detects the changes in light absorption

${ }^{\star}$ Address all correspondence to: Nathan Hervey, E-mail: nhervey@mavs.uta .edu and scattering in tissue caused by changes in concentration of oxyhemoglobin $(\Delta \mathrm{HbO})$ and deoxyhemoglobin $(\Delta \mathrm{Hb})$ secondary to neuronal activity, known as neurovascular coupling. ${ }^{8}$ Although this optical technology is limited to cortical imaging at a modest spatial resolution, it can potentially offer high activation-related signal detection sensitivity at high temporal resolution and a relative robustness to motion artifacts. ${ }^{9}$

Nevertheless, current fNIRS imaging protocols do not typically factor in the variability of subject motions into the image formation process. As a result, when subjects cannot perform the protocol without unintended motions or without activating additional muscles to support the intended motions, as can be the case with $\mathrm{CP},{ }^{10}$ the resulting fNIRS images are contaminated by these additional activation contributions. To better understand sensorimotor cortex plasticity in children with $\mathrm{CP}$ and the response to treatment, it would be desirable to identify and, if possible, separate brain activation patterns due to unintended motions or compensatory muscle activity.

A few recent fMRI studies on adult patients with stroke ${ }^{11}$ and Parkinson's ${ }^{12}$ have employed motion capture technology as a means to improve the sensitivity of detected activation patterns by regressing with the actual patient motions rather than the 
intended ones. Electromyography (EMG) recordings have also been shown to improve fMRI signal analysis outcomes when used as signal regressors for patients with tremors ${ }^{13,14}$ and in healthy adults ${ }^{15}$ as the movements dictated by the activation protocol are never performed perfectly even when the subjects fully intend to do so. Though these studies provide evidence that functional brain image analysis can be improved by use of motion capture and EMG technologies, the challenge of identifying mirror motion contributions in activation images has not been addressed to date.

In this study, a new method is proposed that combines the principles of independent component analysis (ICA) and a general linear model (GLM), henceforth referred to as the GLM/ ICA method, to help identify the contributions of mirror motions in fNIRS images. Specifically, EMG and a multicamera motion tracking system were used synchronously with fNIRS to quantify arm muscle activation and finger motions of children with $\mathrm{CP}$ performing a finger-tapping task. In contrast to the standard practice of using a boxcar function to represent the relative duration of finger tapping, ${ }^{16}$ subject-specific EMG and motion capture data were used instead to create regressors. Data were recorded from the tapping as well as the nontapping hand and were processed by a two-regressor GLM, with one regressor for the tapping and another for the nontapping hand, with the independent components (ICs) decomposed from the $\Delta \mathrm{HbO}$ time-series data by ICA. By identifying which $\Delta \mathrm{HbO}$ ICs uniquely correlated with either tapping or mirror hand motion, two $\Delta \mathrm{HbO}$ images were reconstructed from a single fNIRS data set. One image represented the finger tapping map and the other image represented the unintended mirror motions map. It is shown that these images help shed light on the relative contributions of each hand to cortical sensorimotor center activation and that the pattern of these contributions is unique to each child.

The aforementioned two-regressor GLM/ICA method is not applicable when mirror motions are not present as no regressor, and therefore no corresponding image, can be created for the nontapping hand. Therefore, the two-regressor GLM/ICA method is not applicable to typically developing children who do not exhibit mirror motions. Nevertheless, it is shown that for a typically developing child that performed unintentional extraneous motions with the tapping hand during the activation protocol, a modified one-regressor version of the GLM/ ICA method could provide an improved activation image compared to standard boxcar analysis.

\section{Methods}

\subsection{Subjects}

Eight children with hemiparetic $\mathrm{CP}$ were included in the study (subjects 1 to 8 , six male and two female, $10 \pm 1.7$ years old) along with eight typically developing children as control subjects (five male and three female, $9.5 \pm 1.5$ years old). Each subject performed two tapping trials, one with each hand, making a total of 16 trials for the group of subjects with CP. Subjects 6, 7, and 8 did not exhibit mirror motions while performing the tasks. Excessive head motions and a failure to follow the protocol resulted in unreliable data for subject 5. Additionally, subjects 2 and 4 did not exhibit mirror motions while tapping with their unaffected hand. The GLM/ICA method was applicable to the six remaining trials where mirror motions were present. Three of the subjects were classified as level 2 on the Manual Ability
Classification System (MACS), ${ }^{17}$ two of whom were righthand affected (subjects 2 and 3 ) and one was left-hand affected (subject 1). One subject (subject 4) was classified as MACS level 1 and was right-hand affected. Anatomical T1-MPRAGE MRI images were available for all subjects recruited for this study, which enabled localizing their brain lesions. Subject 1 proved to be a unique case because at seven years of age, due to intractable epileptic seizures, he underwent a right functional peri-insular hemispherectomy involving removal of part of the right temporal lobe, a section of the corpus callosum (disconnection of both hemispheres), and subcortical deafferentation of the right frontal, parietal, and occipital lobes. This subject had a physical shift in the brain midline separating the two hemispheres, as verified by the anatomical MRI, and presented atypical sensorimotor activation patterns that are discussed in this work. These studies were performed under the approval of the University of Texas Southwestern Medical Center at Dallas institutional review board protocol (IRB No. 042007-064).

\subsection{Experimental Protocol and Measurement Setup}

Each subject performed a finger-tapping task, once with their affected hand and once with their nonaffected hand, while the other hand was to remain at rest to the best of the subject's ability. Children were instructed to tap only with four fingers (excluding the thumb) in unison and keep their wrists on the table while finger tapping. A program with an engaging animation made in-house on Adobe Flash (Adobe Systems Incorporated, San Jose, Calfornia) was used to prompt the children to tap at a frequency of $1 \mathrm{~Hz}$. The experimental protocol for finger tapping with each arm consisted of eight 15-s tapping periods, each followed by $25 \mathrm{~s}$ of rest, with a 3-min rest period before the first tapping period. For the motion tracking of hands and arms during the finger tapping task, 5-mm-diameter hemispherical retroreflective targets (B\&L Engineering, Santa Ana, California) were attached with tape on the finger nail bed and just above the proximal interphalangeal joint on each finger, excluding the thumb [Fig. 1(a)]. The three-dimensional coordinates of these retroreflective targets were tracked by a

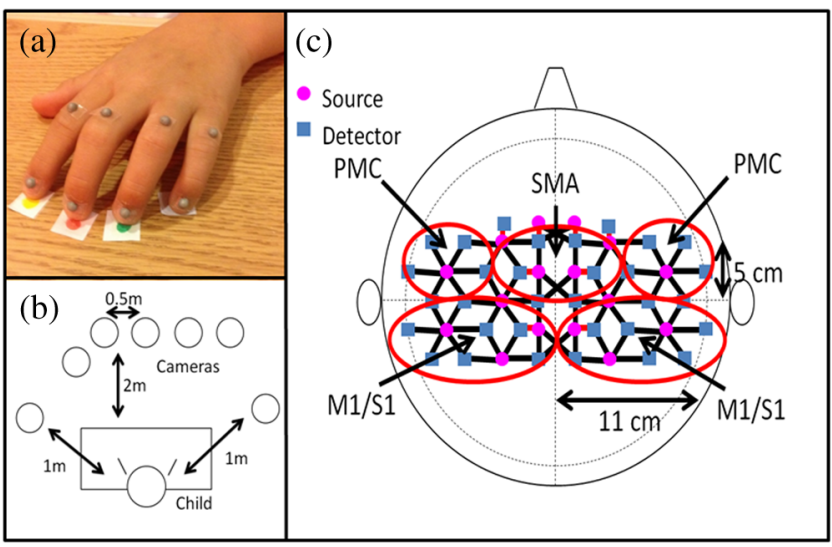

Fig. 1 The motion tracking and functional near-infrared spectroscopy measurement setups: (a) retroreflective target placement on a subject's hand, (b) camera setup relative to the child's position, and (c) location of sources and detectors on the head with covered cortical areas, premotor cortex (PMC), supplementary motor area (SMA), and primary motor/sensory area (M1/S1) circled in red. 
six-camera VICON Mx motion capture system (Vicon, Denver, Colorado) at a sampling rate of $120 \mathrm{~Hz}$. Three of the six analyzed trials were performed with a seven-camera setup shown in Fig. 1(b), where the two cameras to the sides of the subject were $\sim 1.5 \mathrm{~m}$ high, and all others $\sim 3 \mathrm{~m}$ high. The remaining three trials employed a six-camera setup arranged in a semicircle around the subject with a distance of $\sim 3 \mathrm{~m}$ from the subject and heights ranging from 2 to $4 \mathrm{~m}$. The reason that two different camera setups were used in this work was that after subjects 1 and 4 were measured, the study was moved to a different room, which was smaller and camera geometry accommodations had to be made to maintain consistent retroreflective marker tracking. The retroreflective target localization accuracy in both rooms was $\pm 1 \mathrm{~mm}$, which was much smaller than the finger tapping amplitude. More marker occlusions were observed in the smaller room, but due to the high number of markers on each hand, at least one marker provided sufficient trajectory data in any one measurement. Muscle movement was monitored by eight sets of wireless EMG electrodes (BTS FREEEMG 300, BTS, Garbagnate Milanese MI, Italy) placed on the finger flexor and extensor muscles, biceps, and triceps of both arms.

A continuous wave fNIRS system (CW-6, Techen Inc., Milford, Massachusetts) was used to measure $\Delta \mathrm{HbO}$ in the sensorimotor cortex during the finger tapping tasks. A custom headset was created with 16 source fiber bundles [Fig. 1(c), magenta circles] and 32 detector fiber bundles [Fig. 1(c), blue squares]. The headset was centered on the $\mathrm{Cz}$ position of the EEG International 10/20 system $^{18}$ and attached to the subject's head with Velcro straps. Anatomical measurements of the C3, C4, F3, and F4 positions made before each fNIRS session were sufficient to localize the primary motor/sensory area (M1/S1), premotor cortex (PMC), and supplementary motor area (SMA) [Fig. 1(c)]. Each source bundle simultaneously emitted 690 and $830 \mathrm{~nm}$ light, which was received by up to six detectors located within a $3 \mathrm{~cm}$ radius giving a total of 84 source-detector pairs for each wavelength. Eight short $(1.5 \mathrm{~cm})$ source-detector pairs were employed to measure background $\Delta \mathrm{HbO}$ fluctuations in the scalp and used to adaptively filter the fNIRS signals (Sec. 2.4). The detected light signal was sampled at a rate of $25 \mathrm{~Hz}$, and the system utilized frequency modulation (6.4 to $12.6 \mathrm{kHz}$, with $200 \mathrm{~Hz}$ increment) to simultaneously monitor activation over all source-detector pairs for the duration of the trial. Data acquisition for the fNIRS, EMG, and motion tracking systems was manually started simultaneously at the beginning of each measurement protocol, which afforded a synchronicity of $\pm 0.5 \mathrm{~s}$ between modalities that is much shorter than the duration of elicited hemodynamic responses.

\subsection{Signal Processing}

Systemic cerebral hemodynamic fluctuations are present in fNIRS signals due to physiological events, such as cardiac pulsation, respiration, and Mayer waves, which typically fall within 0.8 to $2.0,0.1$ to 0.33 , and $\sim 0.1 \mathrm{~Hz}$ or lower frequency ranges, respectively. ${ }^{19,20}$ Cortical hemodynamic responses to motor task protocols usually fall within the 0.01 to $0.4 \mathrm{~Hz}$ frequency range, so there is overlap between the evoked response and the physiological artifacts of respiration and Mayer waves.

To remove these artifacts, a combination of band-pass and adaptive filtering was used. Cardiac pulsation was removed by band-pass filtering all signals between 0.01 and $0.4 \mathrm{~Hz}$. To remove respiration and Mayer waves, an adaptive least mean square filter was then applied using a short $(1.5 \mathrm{~cm})$ sourcedetector pair as a reference channel as previously reported. ${ }^{21}$ Additionally, data were visually inspected and any tapping/ rest blocks with obvious motion artifacts were excluded from further analysis.

The acquired reflectance data were reconstructed into fNIRS activation images using the open-source HomER software implemented in MATLAB® (Mathworks, Natick, Massachusetts). ${ }^{22}$ HomER uses the Tikhonov perturbation solution to the photon diffusion equation, which employs a regularized MoorePenrose inversion scheme ${ }^{23,24}$ to reconstruct activation images $(20 \times 21$ pixels $)$ that represent maps of $\Delta \mathrm{HbO}$ on the cortical surface within the detectors' field of view [Fig. 1(c)].

\subsection{GLM Analysis with a Boxcar Regressor}

A standard GLM analysis was performed with a boxcar regressor using Eq. (1).

$\boldsymbol{Y}=X_{0} \beta_{0}+X_{1} \beta_{1}+\boldsymbol{\epsilon}$,

where $\boldsymbol{Y}$ represents a column matrix containing the measured hemodynamic response time series, concatenated by pixel $(20 \times 21$ pixels) and $\boldsymbol{\varepsilon}$ represents the model error. The $X$ 's represent the regressors that modeled the expected hemodynamic response. In this case, $X_{0}$ was simply a column of ones that modeled the baseline activation, or direct current (DC) value, and $X_{1}$ was a boxcar function regressor coinciding with the experimental protocol (value of 1 when tapping, 0 when resting) convolved with a hemodynamic response function (HRF). The HRF, which modeled the predicted hemodynamic response per tap, was assumed to be a sum of six gamma functions. ${ }^{25}$ The $\beta$ 's in Eq. (1) represent the unknown predictor coefficients, which were computed using ordinary least squares. ${ }^{26}$ Though generally not true for the low spatial resolution fNIRS images, the GLM method assumed that all pixels and time point data were independent of each other and that their variance was constant. ${ }^{26}$ The $\beta$ estimates per image pixel derived from the regression were used to calculate a $t$ value [Eq. (2)] that determined the image pixels with significant activation.

$t=\frac{c^{\prime} \beta}{\sqrt{\operatorname{Var}(\boldsymbol{\epsilon}) c^{\prime}\left(\boldsymbol{X}^{\prime} \boldsymbol{X}\right)^{-1} c}}$.

All terms in Eq. (2) were introduced in Eq. (1) except for $c$, which represents a contrast vector that allowed for hypothesis testing. A contrast vector of $[-1,1]$ was used to test the null hypothesis that activation was not above baseline, i.e., $\beta_{1}=\beta_{0}$. With the application of the Bonferroni's correction for multiple comparisons, ${ }^{27}$ a pixel with $p<0.0001(|t|>3.5)$ was considered to have significant cortical activity relative to baseline conditions. Activation images were created with the significant $\beta_{1}$ values, which gave $\Delta \mathrm{HbO}$ in $\mu \mathrm{Mol}$. To perform group analysis on the control subjects, the $\boldsymbol{Y}$ matrix in Eq. (1) was created by concatenating the $\Delta \mathrm{HbO}$ time-series data for each subject.

\subsection{GLM/ICA Method}

\subsubsection{Creation of motion regressors}

Two subject-specific motion regressors were created for each trial, one using kinematic data from the tapping hand and the 
other from the mirror hand. Due to the nature of the tapping task, the largest amplitude changes were seen in the distal markers located on the finger nails [Fig. 1(a)] in the vertical direction relative to the table surface. For this reason, only the vertical trajectories from the distal markers were used to create the regressors. Continuous motion capture data were required for the analysis, but were not always available for all markers. All distal markers with continuous trajectories were used (ranging from 1 to 4), while trajectories with incomplete data due to target occlusions were excluded. In cases where no markers had continuous data due to occlusions but only small gaps in the data stream were present $(<0.5 \mathrm{~s})$, gaps were filled using the MATLAB ${ }^{\circledR}$ function spline, which performs cubic spline interpolation. The selected trajectories were then low-pass filtered at $7 \mathrm{~Hz}$, and the marker velocities were calculated by finding the absolute difference in amplitude for consecutive time points. The resulting velocity data retained the characteristics of the motions while removing drifts present in the displacement trajectories due to children not returning their hand to the table between taps, or changing the position of their wrists. To account for movement variations between the fingers, principal component analysis was applied, and the first principal component was selected and down-sampled from 120 to $25 \mathrm{~Hz}$ to match the fNIRS system sampling rate. Finally, the processed motion capture data were convolved with the HRF.

Kinematic regressors were also created using EMG signals. The signal from the finger extensor muscle from each arm was used to make the corresponding tapping and mirror hand regressors. These signals were band-pass filtered between 20 and $200 \mathrm{~Hz}$ and then full-wave rectified and low-pass filtered at $6 \mathrm{~Hz}$, giving a linear envelope of the muscle activity, with each envelope representing an individual tap. The signals were then down-sampled from 1000 to $25 \mathrm{~Hz}$ to match the fNIRS sampling rate and convolved with the HRF.

\subsubsection{Independent component analysis}

ICA aims to separate recorded signals, which are assumed to be a mixture of source signals, into the signals originally produced by the sources. The ICA equation is described by

$x=\boldsymbol{A} s$,

where $\boldsymbol{A}$ is the mixing matrix, $s$ is a vector containing the sources, and $x$ is a vector containing the measured mixed signals. All ICA in this study was performed with the FastICA algorithm provided online by researchers at the University of Helsinki. ${ }^{28}$

For all analyses, the optional parameter for nonlinearity was set to skew, 10,000 was used for the maximum number of iterations, and all other parameters were left at the default values. The algorithm allows the user to specify the number of ICs to be found. However, when too many are selected, the algorithm does not converge. The FastICA algorithm was run on the reconstructed $\Delta \mathrm{HbO}$ time-series data $(21 \times 20$ pixels $)$ several times to find the maximum number of ICs supported by each data set, which was between 23 and 32 .

\subsubsection{Selection of significant ICs using a motion regressor GLM}

Due to the stochastic nature of the FastICA algorithm, some ICs were found in every run, while others were not. To determine which ICs belonged to the consistently appearing set, an initial run of the FastICA algorithm was performed on the $\Delta \mathrm{HbO}$ time-series data and the components produced were saved for use in the subsequent steps. Next, the FastICA algorithm was run 30 times with each run comparing its ICs to the initial set of ICs. Because the ICA is stochastic, it produced the ICs in a different order each time it was run. Therefore, each IC in any one run had to be matched to the IC of the first run. As the ICs within any one set were statistically independent, each IC in the first run could be uniquely matched to the corresponding IC of any subsequent run by calculating their pairwise correlation coefficient (CC). Only ICs with average CC $>0.9$ over the 30 runs were used in subsequent steps, as it was empirically determined that lower average $\mathrm{CC}$ values corresponded to ICs not found in every run.

To determine if a tapping or mirror motion regressor explained the ICs, a GLM analysis was performed using

$\boldsymbol{Y}=X_{0} \beta_{0}+X_{1} \beta_{1}+X_{2} \beta_{2}+X_{1} X_{2} \beta_{3}+\boldsymbol{\epsilon}$,

where $\boldsymbol{Y}$ represents the matrix containing the ICs, $X_{1}$ and $X_{2}$ represent the motion tracking or EMG regressors for the tapping and mirror hand, respectively, $\beta_{1}, \beta_{2}$, and $\beta_{3}$ were the corresponding scaling factor model estimates, and $X_{0}$ and $\boldsymbol{\varepsilon}$ were the DC and error terms as in Eq. (1). The $X_{1} X_{2}$ term was included as a statistical interaction term to reduce the amount of cross-talk in the model as the tapping and mirror hand motion regressors were usually correlated to varying degrees. $t$ values were calculated with Eq. (2) using contrasts of $c=[-1,1,0,0]$ and $c=[-1,0,1,0]$ to test whether the tapping or mirror hand regressors, respectively, explained a significant portion of the ICs. Note that if no mirror motions are present, the $X_{2}$ regressor is zero and the GLM analysis collapses into a one-regressor model, which cannot provide two images corresponding to the tapping and mirror hand motions. However, a modified GLM/ICA can be performed using only one motion regressor by excluding the mirror and cross-talk regressors from the analysis. The difference between the one-regressor GLM/ICA and the boxcar method is that the former convolves the hemodynamic response function with the profile of the actual motions of the tapping hand, including any unintended motions during rest periods, to generate a more accurate model predicting the observed hemodynamic fluctuation data.

The GLM analysis in Eq. (4) was performed on each of the 30 FastICA runs mentioned above. Therefore, each IC had a group of $30 t$ values for the tapping and 30 for the mirror regressor associated with it. To determine whether the tapping or mirror hand motion regressor explained a significant portion of a given IC, two right-sided one sample $t$ tests were performed $(\alpha=0.05)$ on the saved tapping and mirror regressor $t$ values, respectively, for each IC. If the null hypothesis $|t|<$ 3.5 (see Sec. 2.5 for selection of $t$ value) was rejected for a set of $t$ values, the corresponding regressor was considered to explain a significant amount of the variation for that IC. The ICs significantly explained by both regressors were considered as cross-talk and were discarded along with those explained by neither regressor. If an IC was significantly explained by one regressor and not the other, it was saved for the subsequent step of $\Delta \mathrm{HbO}$ activation image formation for that regressor. 


\subsubsection{Creation of activation images for tapping and mirror motions}

As the ICA analysis does not take into account the sign of the ICs, this was double-checked to be consistent with the majority of the FastICA runs. Subsequently, the $\Delta \mathrm{HbO}$ time-series activation images for the tapping and mirror regressor groups were reconstructed individually using

$\boldsymbol{Y}_{\mathrm{ICA}}=\boldsymbol{A} * \boldsymbol{C} * \boldsymbol{X}$,

where $\boldsymbol{Y}_{\text {ICA }}$ is the matrix containing the $\Delta \mathrm{HbO}$ time series for each pixel, $\boldsymbol{A}$ is the mixing matrix saved from Eq. (3), $\boldsymbol{X}$ is the matrix of ICs, and $\boldsymbol{C}$ is a square matrix with ones at positions corresponding either to all ICs from the tapping or mirror motions and zeroes elsewhere. Two activation images, one for each motion, were created individually using the GLM approach described in Sec. 2.5, but with $\boldsymbol{Y}$ and $X_{1}$ from Eq. (1) replaced with new terms. For the tapping image, $\boldsymbol{Y}$ from Eq. (1) was replaced with the $\boldsymbol{Y}_{\mathbf{I C A}}$ reconstructed from the ICs corresponding to the tapping motions, and $X_{1}$ from Eq. (1) was replaced with tapping hand motion regressor $\left[X_{1}\right.$ in Eq. (4)]. Similarly, the mirror image was created by replacing $\boldsymbol{Y}$ from Eq. (1) with the $\boldsymbol{Y}_{\text {ICA }}$ reconstructed from the mirror ICs and $X_{1}$ from Eq. (1) with the mirror hand motion regressor $\left[X_{2}\right.$ in Eq. (4)].

\section{Results}

\subsection{Identification of Brain Activation Areas due to Tapping and Mirror Motions Using Motion Tracking}

\subsubsection{Determination that activation in M1/S1 seen in the boxcar image was due to tapping after removal of activation from the mirror image}

The GLM/ICA method was first applied to subject 1 performing a tapping task with his unaffected right hand. Figures 2(a) and 2(b) show the vertical displacement recorded from the index finger distal target on the right and left hand, respectively. Figure 2(b) indicates that mirror motions were present during this trial, but they were lower in amplitude and sparser than the tapping hand motions seen in Fig. 2(a). Displacements were qualitatively similar in all other trials and are, therefore, not shown in subsequent figures. Figures 2(c) and 2(d) show the tapping (right hand) and mirror (left hand) images, respectively, produced by the GLM/ICA method. Figure 2(e) shows the activation image created using the standard boxcar regressor analysis for the right hand tapping trial, and Fig. 2(f) shows the standard boxcar image for subject 1's left hand tapping trial. Note that in Figs. 2(c) to 2(f) and all subsequent figures, a dashed black line indicates the midline and gray color ovals indicate the locations of M1/S1, PMC functional areas in each
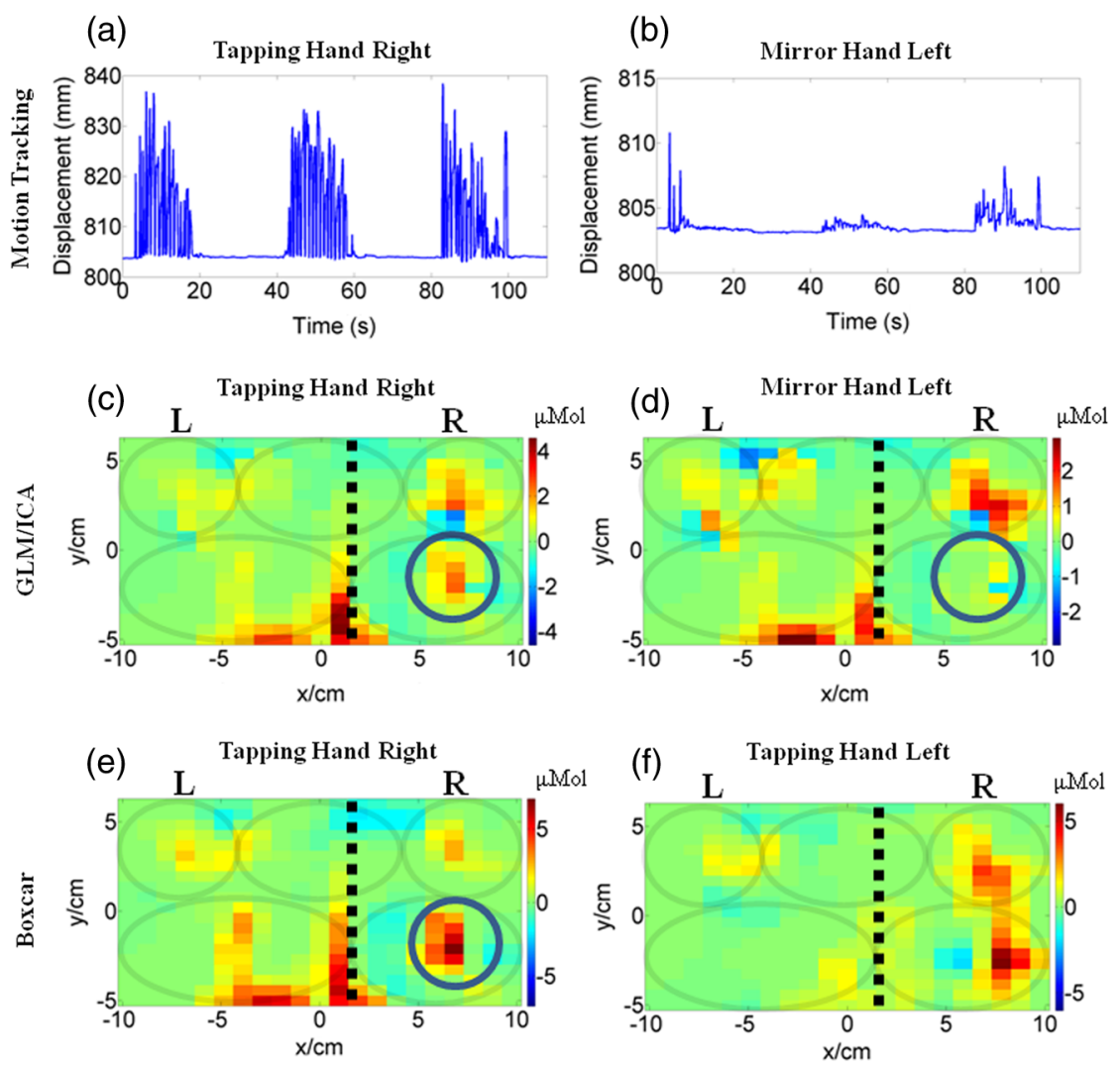

Fig. 2 Comparison of activation images made with the general linear model/independent component analysis (GLM/ICA) method or a boxcar regressor for subject 1 performing right hand tapping. (a) Vertical position of nail target on tapping (right) hand and (b) on the mirror (left) hand. (c) Image created using the GLM/ICA method for the tapping (right) hand and (d) the mirror (left) hand. (e) Activation image made using a boxcar regressor for right hand tapping. (f) Activation image made using a boxcar regressor for left hand tapping. The black dashed lines indicate the shifted midline and the gray ovals indicate the M1/S1 and PMC of each hemisphere and the SMA shared between hemispheres. 
brain hemisphere, and SMA shared between hemispheres, as described in Sec. 2.2 and Fig. 1(c) above. Comparing Fig. 2(c) to Fig. 2(d) revealed that the area of activation present in the right primary $\mathrm{M} 1 / \mathrm{S} 1$ area of the tapping hand image [Fig. 2(c)] was absent from the mirror hand image [Fig. 2(d)], as indicated by the circles, but the images were otherwise similar. Overlap was seen in the right and left PMC areas where activation was present in both images [Figs. 2(c) and 2(d)], as well as the midline activation areas. These midline activations, which are atypical of hand tapping tasks, were specific to this subject and likely due to the shift in the left brain hemisphere mentioned in Sec. 2.1. Despite the spatial overlap of several activation areas between tapping and mirror images [Figs. 2(c) and 2(d)], the GLM/ICA method provided an advantage as it removed some ambiguity present in the standard boxcar image [Fig. 3(e)] that could not attribute any activation area solely to one type of motion. The method was also applied to subject 1's left hand tapping task and similar results were obtained (not shown for brevity). It was interesting to see that the M1/S1 and part of the PMC areas activated for this subject during left hand tapping were slightly displaced relative to the corresponding areas activating for right hand tapping [compare Fig. 2(e) to Fig. 2(f) boxcar images].

\subsubsection{Determination that activation in M1/S1 seen in the boxcar image was due to mirror motions after removal of activation from the tapping image}

The GLM/ICA method was next applied to data from subject 2 performing right (affected) hand tapping. Figures 3(a) and 3(b) show the tapping (right hand) image and mirror (left hand) images produced by the GLM/ICA method, and Figs. 3(c) and 3(d) show the standard boxcar images for the right hand and left hand trials, respectively. Notice that activation in the ipsilateral (right) M1/S1 area, indicated by circles, was present in both the boxcar and mirror images [Figs. 3(c) and 3(b)], but was relatively weak in the tapping image [Fig. 3(a)]. This suggests that activation in the right M1/S1 area was primarily due to mirror (left hand) motions, as would be physiologically expected. This conclusion was further supported by the fact that in subject 2's left (unaffected) hand tapping trial, where no mirror motions were present, the right M1/S1 area showed strong activation as seen in Fig. 3(d). In this case, the GLM/ICA method was able to clean up the tapping activation image by removing mirror motion contamination and, thus, provide superior results to the standard boxcar method.

\subsubsection{Determination that bilateral activation in M1/S1 seen in the boxcar image was due to tapping in one brain hemisphere and due to mirror motions in the other}

The results of the GLM/ICA method applied to subject 3 performing a right (affected) hand tapping task are shown in Fig. 4. Figures 4(a) and 4(b) show the tapping (right hand) and mirror (left hand) activation images, respectively, and Figs. 4(c) and 4(d) show the boxcar images for the right and left hand trials, respectively. Notice that similar to the results for subject 2 (Sec. 3.2.2), the activation in the ipsilateral (right) primary M1/S1 area (red circles in Fig. 4) present in the right hand boxcar image [Fig. 4(c)] was mostly removed from the tapping image [Fig. 4(a)]. In addition, an activation area in the left SMA (gray circles in Fig. 4) was also removed from the tapping image [Fig. 4(a)]. Both areas were assigned to the mirror hand image [Fig. 4(b)], indicating that mirror (left hand) motions were primarily responsible for activation in these areas.

In addition, activation in the contralateral (left) M1/S1 area (blue circles in Fig. 4) was absent in the mirror image [Fig. 4(b)], but was present in the tapping and boxcar images [Figs. 4(a) and 4(c)]. In this case, the GLM/ICA method provided another cleaned-up activation image by removing contamination from mirror motions, demonstrating its superiority over standard boxcar analysis.
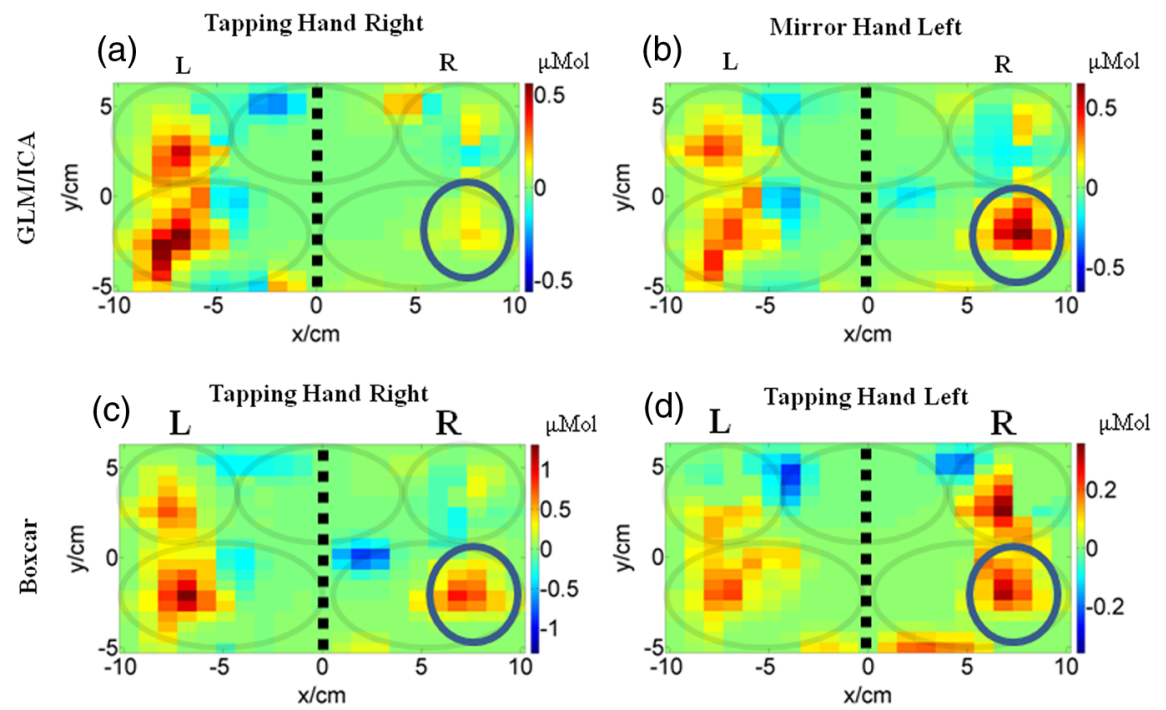

Fig. 3 Comparison of activation images made with the GLM/ICA method or a boxcar regressor for subject 2 performing right hand tapping. (a) Image created using GLM/ICA for the tapping (right) hand and (b) the mirror (left) hand. (c) Activation image made using a boxcar regressor for right hand tapping. (d) Activation image made using a boxcar regressor for left hand tapping. The black dashed lines indicate the midline and the gray ovals indicate the M1/S1 and PMC of each hemisphere and the SMA shared between hemispheres. 


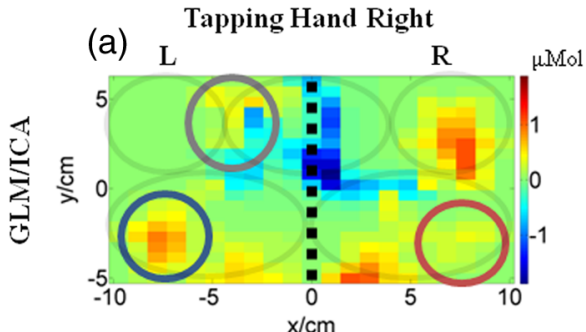

(c)

Tapping Hand Right

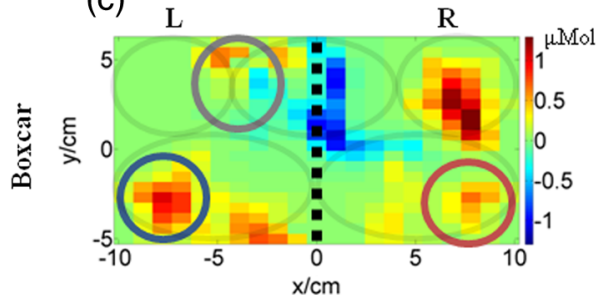

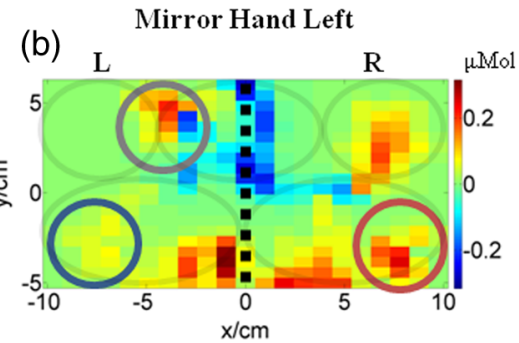

Tapping Hand Left

(d)

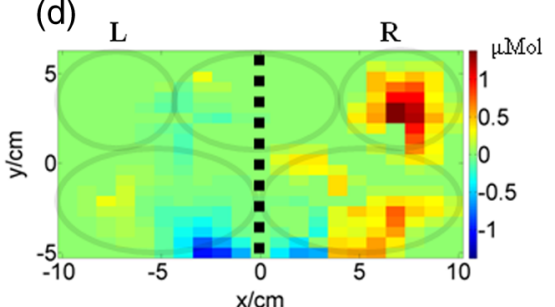

Fig. 4 Comparison of activation images made with the GLM/ICA method and a boxcar regressor for subject 3 performing right hand tapping. (a) Image created using the GLM/ICA method for the tapping (right) hand and (b) the mirror (left) hand. (c) Activation image made using a boxcar regressor for right hand tapping. (d) Activation image made using a boxcar regressor for left hand tapping. The black dashed lines indicate the midline and the gray ovals indicate the M1/S1 and PMC of each hemisphere and the SMA shared between hemispheres.

Subject 3 also exhibited mirror motions while tapping with his left (unaffected) hand, and Fig. 4(d) shows the boxcar image from this trial containing contralateral M1/S1 and PMC activation, similar to Fig. 4(b) for right hand tapping. The GLM/ICA results of this trial are not shown, because the method produced tapping and mirror images that looked nearly identical and, therefore, did not provide any useful information. This occurred because subject 3's right (affected) hand mirror motions were highly correlated (0.93) with the left (unaffected) hand tapping motions. The effect of motion correlation on GLM/ICA results is further discussed in Sec. 4.4.

\subsection{Identification of Brain Activation Areas due to Tapping and Mirror Motions Using EMG}

The GLM/ICA method was applied to subject 4 performing a right (affected) hand tapping task, but, unlike all other cases, the motion regressors were created using EMG signals. The reason was that subject 4 had no detectable mirror (left hand) motions, so no motion tracking regressors could be created. However, muscle activity was detected in the mirror (left) hand, indicating the subject was pressing the mirror (left) arm against the table while finger tapping with the other (right) hand. Figures 5(a) and
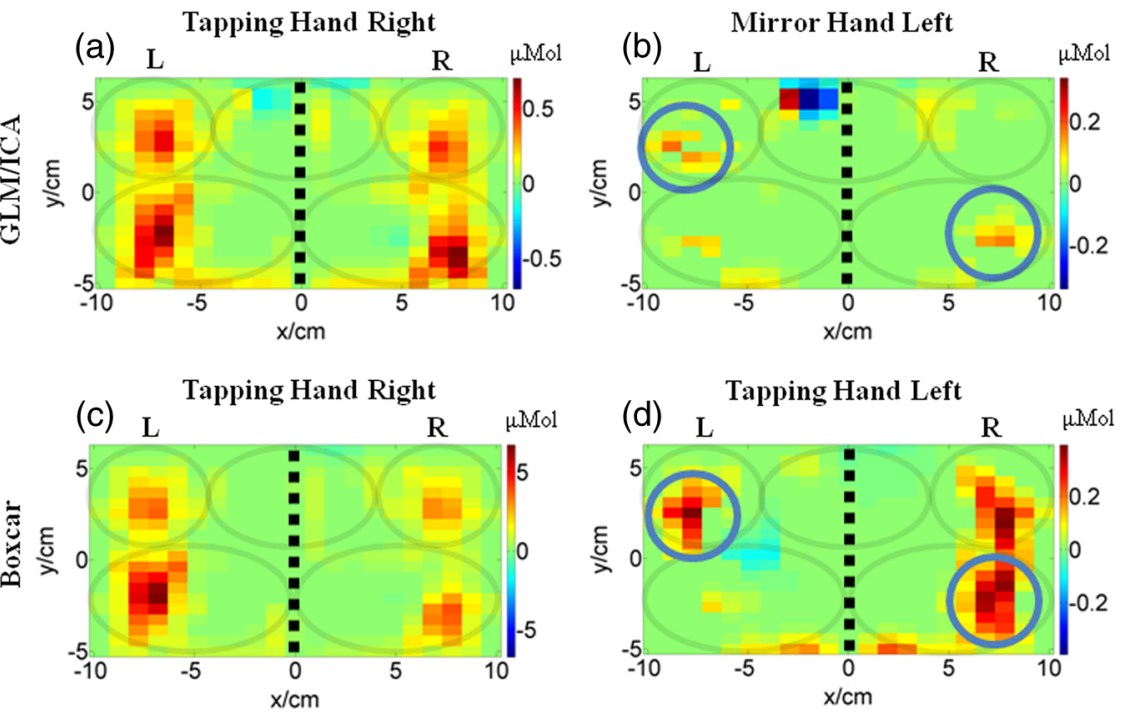

Fig. 5 Comparison of activation images made with the GLM/ICA method and a boxcar regressor for subject 4 performing right hand tapping. (a) Image created using GLM/ICA method for the tapping (right) hand and (b) the mirror (left) hand. (c) Activation image made using a boxcar regressor. (d) Activation image made using a boxcar regressor for left hand tapping. The black dashed lines indicate the midline and the gray ovals indicate the M1/S1 and PMC of each hemisphere and the SMA area shared between hemispheres. 
5(b) show the tapping (right hand) and mirror (left hand) images, respectively. Figures $5(\mathrm{c})$ and $5(\mathrm{~d})$ show the corresponding boxcar images for the right and left hand trials, respectively. In this case, the boxcar [Fig. 5(c)] and tapping images [Fig. 5(a)] looked similar, with M1/S1 and PMC areas activating bilaterally during the finger tapping motion. Bilateral activation in sensorimotor areas is common in children with $\mathrm{CP}$, but is not seen in all cases as activation patterns are variable in this population. ${ }^{29,30}$ Interestingly, in the mirror hand image [Fig. 5(b)], the ipsilateral M1/S1 and contralateral PMC showed weak levels of activation consistent with the relatively sparse and low-amplitude mirror hand muscle activations. These areas were also active during the left hand tapping trial, but their size was larger and also involved activation of the ipsilateral (left) PMC [Fig. 5(d)]. The observed differences between the mirror hand image [Fig. 5(b)] and the left hand tapping one [Fig. 5(d)] were not surprising since these two activation images were not generated by the same type of motion. Nevertheless, Fig. 5(b) suggests that when the mirror hand was pressing against the table, a relatively small contribution was added to the activation image seen by boxcar analysis [Fig. 5(c)], though the latter would not have identified the origin of these mirror hand contributions.

\subsection{Application of a One-Regressor GLM/ICA Method to a Typically Developing Child}

The modified one-regressor GLM/ICA method was applied to the right hand trial of subject 9 , who was a typically developing child. Figures 6(a) and 6(b) show the images created using the standard boxcar analysis and the modified one-regressor GLM/ ICA method, respectively. Figure 6(c) shows the vertical displacement of the marker placed on the nail of the tapping index finger. The red arrows in Fig. 6(c) indicate that subject 9 moved during the resting portions of the protocol (only three tapping intervals are shown for clarity). Figure 6(d) shows the group analysis performed using the right hand tapping trials from six right-handed control subjects. Two of the eight control subjects were excluded from the group analysis, one who was left handed and one due to motion artifacts. The three circles in Figs. 6(a) and 6(b) indicate areas where the activation patterns differed in the two images. In the contralateral (left) M1/S1 area (blue circles), activation was increased in the GLM/ICA image [Fig. 6(b)], while in the contralateral (left) PMC, it was decreased (red circles). Additionally, an area of deactivation was increased in the ipsilateral (right) M1/S1 area (gray circles) in the GLM/ICA image [Fig. 6(b)]. In all three areas, the GLM/ICA image [Fig. 6(b)] more closely matched the group analysis image [Fig. 6(d)] than the boxcar image [Fig. 6(a)]. In this case, the GLM/ICA method provided an improved image by taking into account the unintended motions occurring during the rest periods. In the more typical case where a subject followed the protocol correctly without extraneous motions, no significant differences were seen between the boxcar and GLM/ICA images.

\section{Discussion}

\subsection{Overview}

A shortcoming of current standard GLM analyses for fNIRS data is that they cannot distinguish whether sensorimotor cortex areas activate due to intended or unintended mirror motions. In this study, the GLM/ICA method was developed to help address this shortcoming. The method was applied to fNIRS data acquired during a hand tapping task performed by children with $\mathrm{CP}$ who exhibited unintended mirror motions. In some cases (subjects 2 and 3), activation areas present in the standard boxcar image were removed from the tapping hand image, thus providing a cleaned-up activation image with mirror motions contamination removed. In other cases (subjects 1 and 4), the
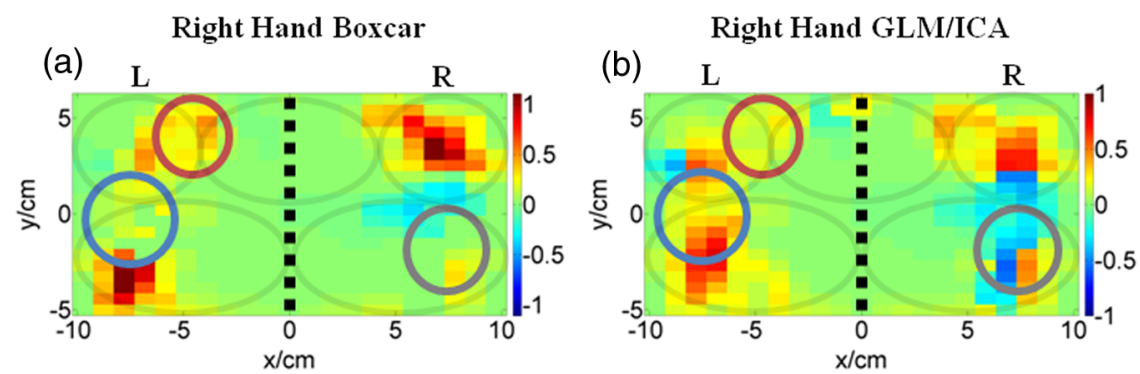

(c) Right Hand Displacement

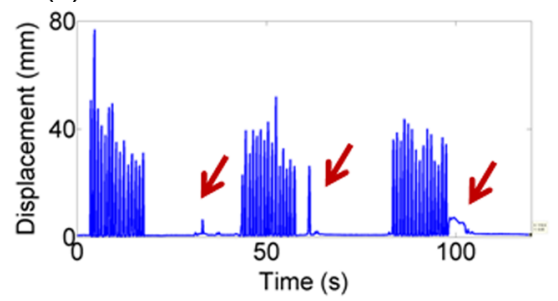

Right Hand Group Analysis

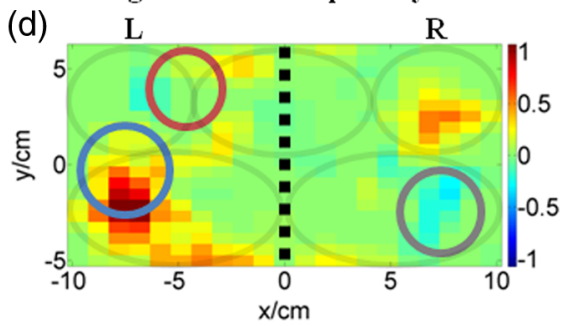

Fig. 6 Comparison of activation images made with the GLM/ICA method and a boxcar regressor for control subject 9 performing right hand tapping. (a) Activation image made using a boxcar regressor. (b) Activation image made with the modified GLM/ICA method. (c) Vertical position of nail target on tapping (right) hand. (d) Group analysis including six typically developing children as control subjects. The black dashed lines indicate the midline and the gray ovals indicate the M1/S1 and PMC of each hemisphere and the SMA shared between hemispheres. 
tapping hand image was not very different from the boxcar one, but the mirror image offered additional information on how unintended motions were mapped onto the sensorimotor cortex. Regardless of which case was achieved, the GLM/ICA method produced additional interesting information that was not attainable by standard boxcar regressor analysis.

\subsection{Special Case of a Subject with Prior Brain Surgery}

The special case of subject 1 merits further discussion. As mentioned in Sec. 2.1, subject 1 had prior brain surgery that resulted in a physical shift of his brain's midline, as verified by anatomical MRI. fNIRS imaging for this subject indicated bilateral activation for tapping with either hand though, interestingly, the activation region in the right M1/S1 was in slightly different locations depending on which hand was tapping. Specifically, during left hand tapping by subject 1 [Fig. 2(f)], an adjacent but distinct activation area was located laterally and posterior to the right hand tapping activation in right M1/S1 seen in Figs. 2(c) and 2(e). The fact that portions of both the right and left M1/S1 areas activated for tapping performed by either hand likely caused each hand to act as the mirror one when the other hand was tapping. For this special case, the GLM/ICA method was especially useful in disentangling the relative contributions of tapping versus mirror motion in this subject with complicated cortical reorganization after surgery.

\subsection{Nonseparable Activation Areas and Motion Regressor Correlation}

In all cases, there were some activation areas that appeared in both the tapping and mirror images, and therefore, the source of these activations remained ambiguous. Since the GLM/ICA method assigned ICs uniquely to the tapping or mirror images, the temporal hemodynamic patterns in these regions were different even though they were spatially overlapping. It is, therefore, possible that in some cases both tapping and mirror motions did contribute to activation in the same areas as plasticity after brain injury may have resulted in one brain area eliciting motion in both hands. ${ }^{31}$ However, it is also possible that some of the activation attributed to the same pixel locations in both images was due to only tapping or mirror motions and that the GLM/ICA method could not identify the responsible motion. This is because the tapping and mirror hand regressors were typically correlated to varying degrees. When two highly correlated regressors were used in the GLM analysis, the model could not uniquely assign the variance in the $\Delta \mathrm{HbO}$ signals to one of the regressors, as applying a high weight to one regressor and low weight to the other produced a solution nearly identical to the opposite weight assignments. This could have resulted in false overlap of activation areas between the two images. In the case of subject 3's left hand tapping trial (Sec. 3.1.3), where the correlation coefficient between motion capture regressors for tapping and mirror motions was high (0.93), the overlap was so severe that the images looked identical and no activation areas could be attributed to only one motion (not shown for brevity). However, this high correlation occurred in only one out of the five motion tracking data sets analyzed in this study. In the remaining four motion tracking data sets, the fraction of ICs rejected because they correlated with both regressors was in the 25 to $50 \%$ range, corresponding to correlation coefficients in the 0.59 to 0.78 range. In all four cases, meaningful information on mirror motion effects was attained despite this limitation.

\subsection{Motion Tracking Versus EMG Regressors}

Since EMG systems are less expensive and require less setup time than a multicamera motion capture system, a valid question would be why use motion capture data when EMG signals could be used instead? The answer is that motion regressors created from EMG signals tended to be more correlated than motion capture data, with correlation coefficients in the 0.72 to 0.96 range. As discussed in Sec. 4.4, higher levels of correlation between the motion regressors resulted in less meaningful results. In all cases where both EMG and motion capture regressors were available, the EMG regressors had higher correlation coefficients than their corresponding motion capture regressors and were, therefore, inferior. However, in the unique case of subject 4 (Sec. 3.2), the EMG regressors had a very low correlation coefficient (0.08), so meaningful results could be obtained.

\subsection{Data Acquisition Limitations}

In addition to the limitation of high correlation between the regressors (Sec. 4.4), this method is limited by issues common to all fNIRS and motion capture studies. In this work, motion artifacts were observed in several data sets. However, in the five subjects analyzed, motion artifacts did not cause any trials to be excluded. Also, motion capture data were not always reliable as occlusions sometimes occurred during the hand tapping task and the children occasionally moved their hands outside the camera system's field of view. Fortunately, this did not occur in any trials for the five subjects analyzed in this study, but only in those excluded due to other reasons, such as the absence of mirror motions.

\section{Conclusion}

In this work, a new GLM/ICA method was proposed for the analysis of fNIRS data acquired during a finger tapping task where unintentional mirror motions were present. Custom regressors were created from motion tracking or EMG data, and fNIRS signals were unmixed by applying ICA to the $\Delta \mathrm{HbO}$ time-series data. A GLM regression was performed with the subject-specific regressors and the ICs significantly explained by one motion regressor and not the other were used to create sensorimotor activation maps corresponding to each type of motion. The method provided additional information not attainable with the standard boxcar method and, therefore, may contribute to an improved understanding of plasticity in children with $\mathrm{CP}$ who exhibit mirror motions as long as these are not highly correlated to the tapping hand motions. The GLM/ICA method is potentially applicable to conditions other than $\mathrm{CP}$, e.g., stroke, and could also be useful in assessing changes in the mirror hand contributions to sensorimotor activation maps as a result of a therapeutic intervention. It was also shown that a modified one-regressor GLM/ICA model could provide superior results compared to the standard boxcar analysis even for control subjects who unintentionally do not accurately follow the experimental protocol.

\section{Acknowledgments}

Support for this work was provided in part by the National Institute of Biomedical Imaging and Bioengineering, Grant No. 1R01EB013313-01. 


\section{References}

1. M. V. Johnston and A. H. Hoon Jr., "Cerebral palsy," Neuromol. Med. 8(4), 435-450 (2006).

2. K. Cahill-Rowley and J. Rose, "Etiology of impaired selective motor control: emerging evidence and its implications for research and treatment in cerebral palsy," Dev. Med. Child Neurol. 56(6), 522-528 (2014).

3. J. P. Phillips et al., "Ankle dorsiflexion fMRI in children with cerebral palsy undergoing intensive body-weight-supported treadmill training: a pilot study," Dev. Med. Child Neurol. 49(1), 39-44 (2007).

4. F. Pilato et al., "Unaffected motor cortex remodeling after hemispherectomy in an epileptic cerebral palsy patient. A TMS and fMRI study," Epilepsy Res. 85(2-3), 243-251 (2009).

5. A. Van de Winckel et al., "How does brain activation differ in children with unilateral cerebral palsy compared to typically developing children, during active and passive movements, and tactile stimulation? An fMRI study," Res. Dev. Disabil. 34(1), 183-197 (2013).

6. W. Yun et al., "Effect of acupuncture on the brain in children with spastic cerebral palsy using functional neuroimaging (fMRI)," J. Child Neurol. 23(11), 1267-1274 (2008).

7. S. K. Piper et al., "A wearable multi-channel fNIRS system for brain imaging in freely moving subjects," NeuroImage 85(Pt 1), 64-71 (2014).

8. M. A. Franceschini et al., "Hemodynamic evoked response of the sensorimotor cortex measured noninvasively with near-infrared optical imaging," Psychophysiology 40(4), 548-560 (2003).

9. F. Orihuela-Espina et al., "Quality control and assurance in functional near infrared spectroscopy (fNIRS) experimentation," Phys. Med. Biol. 55(13), 3701-3724 (2010).

10. J. P. Kuhtz-Buschbeck et al., "Quantitative assessment of mirror movements in children and adolescents with hemiplegic cerebral palsy," Dev. Med. Child Neurol. 42(11), 728-736 (2000).

11. C. Casellato et al., "Simultaneous measurements of kinematics and fMRI: compatibility assessment and case report on recovery evaluation of one stroke patient," J. Neuroeng. Rehabil. 7, 49 (2010).

12. S. Holiga et al., "Accounting for movement increases sensitivity in detecting brain activity in Parkinson's disease," PLoS One 7(5), e36271 (2012).

13. M. F. Contarino et al., "Is there a role for combined EMG-fMRI in exploring the pathophysiology of essential tremor and improving functional neurosurgery?," PLoS One 7(10), e46234 (2012).

14. A. F. van Rootselaar et al., "Simultaneous EMG-functional MRI recordings can directly relate hyperkinetic movements to brain activity," Hum. Brain Mapp. 29(12), 1430-1441 (2008).

15. B. J. MacIntosh et al., "Improving functional magnetic resonance imaging motor studies through simultaneous electromyography recordings," Hum. Brain Mapp. 28(9), 835-845 (2007).
16. M. M. Plichta et al., "Model-based analysis of rapid event-related functional near-infrared spectroscopy (NIRS) data: a parametric validation study," Neuroimage 35(2), 625-634 (2007).

17. A. C. Eliasson et al., "The manual ability classification system (MACS) for children with cerebral palsy: scale development and evidence of validity and reliability," Dev. Med. Child Neurol. 48(7), 549-554 (2006).

18. A. Villringer and B. Chance, "Non-invasive optical spectroscopy and imaging of human brain function," Trends Neurosci. 20(10), 435-442 (1997).

19. M. A. Franceschini et al., "Diffuse optical imaging of the whole head," J. Biomed. Opt. 11(5), 054007 (2006).

20. C. Julien, "The enigma of Mayer waves: facts and models," Cardiovasc. Res. 70(1), 12-21 (2006).

21. B. Khan et al., "Improving optical contact for functional near infrared brain spectroscopy and imaging with brush optodes," Biomed. Opt. Express 3(5), 878-898 (2012).

22. T. J. Huppert et al., "HomER: a review of time-series analysis methods for near-infrared spectroscopy of the brain," Appl. Opt. 48(10), D280D298 (2009).

23. B. Brooksby et al., "Spectral priors improve near-infrared diffuse tomography more than spatial priors," Opt. Lett. 30(15), 1968-1970 (2005).

24. A. Li et al., "Reconstructing chromosphere concentration images directly by continuous-wave diffuse optical tomography," Opt. Lett. 29(3), 256-258 (2004).

25. M. A. Lindquist et al., "Modeling the hemodynamic response function in fMRI: efficiency, bias and mis-modeling," Neuroimage 45(1 Suppl), S187-198 (2009).

26. N. A. Lazar, The Statistical Analysis of Functional MRI Data, SpringerVerlag, New York (2008).

27. A. K. Singh and I. Dan, "Exploring the false discovery rate in multichannel NIRS," Neuroimage 33(2), 542-549 (2006).

28. A. Hyvarinen, "Fast and robust fixed-point algorithms for independent component analysis," IEEE Trans. Neural Netw. 10(3), 626-634 (1999).

29. A. Van de Winckel et al., "How does brain activation differ in children with unilateral cerebral palsy compared to typically developing children, during active and passive movements, and tactile stimulation? An fMRI study," Res. Dev. Disabil. 34(1), 183-197 (2013).

30. M. Weinstein et al., "Interhemispheric and intrahemispheric connectivity and manual skills in children with unilateral cerebral palsy," Brain Struct. Funct. 219(3), 1025-1040 (2014).

31. L. J. Carr, "Development and reorganization of descending motor pathways in children with hemiplegic cerebral palsy," Acta Paediatr. Suppl. 85(Suppl. 416), 53-57 (1996).

Biographies of the authors are not available. 